Florian Straube; Frank Schultz; Michael Makarski; Stefan Weinzierl

\title{
Mixed Analytical-Numerical Filter Design for Optimized Electronic Control of Line Source Arrays
}

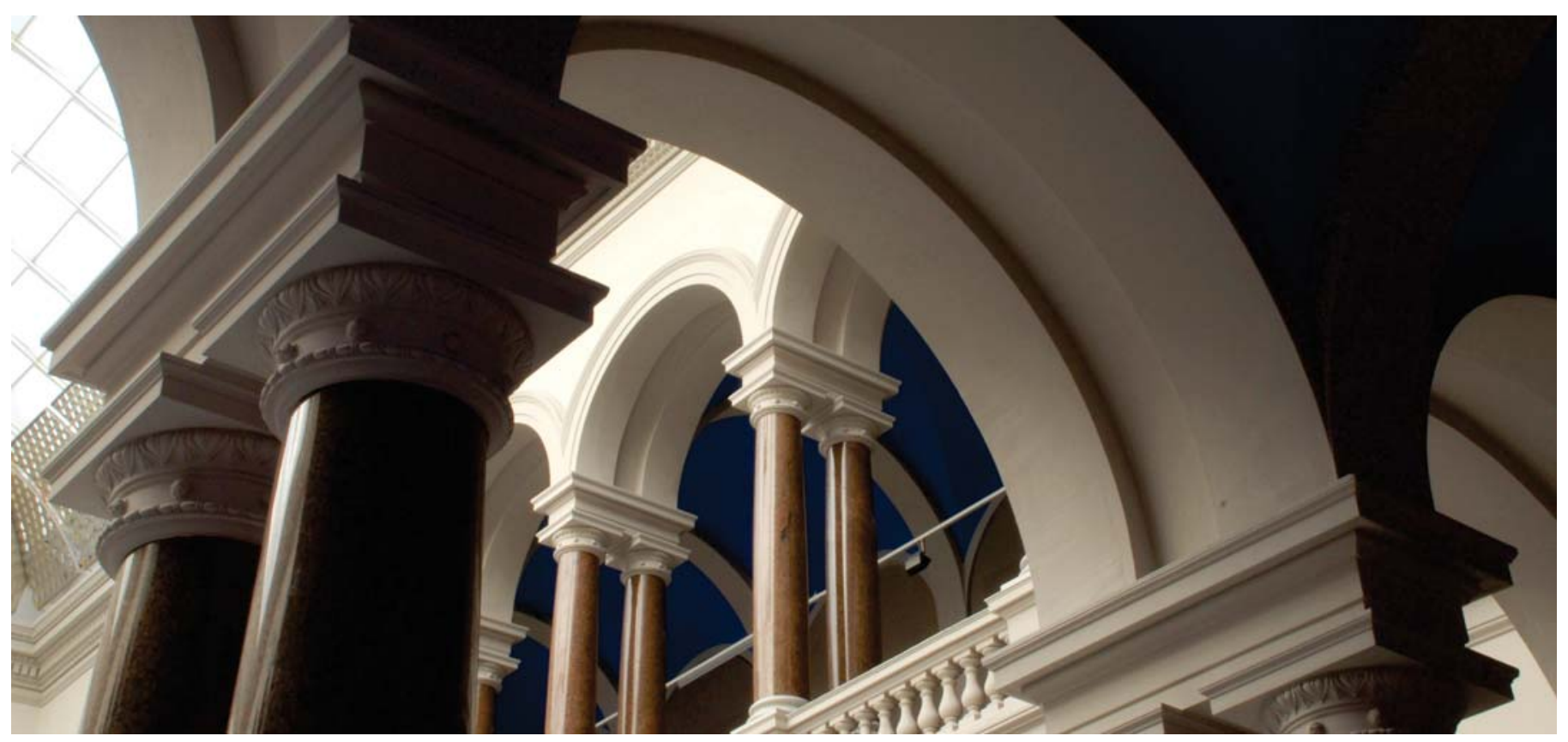

Straube, F., Schultz, F., Makarski, M., \& Weinzierl, S. (2018). Mixed Analytical-Numerical Filter Design for Optimized Electronic Control of Line Source Arrays. Journal of the Audio Engineering Society, 66(9), 690702. https://doi.org/10.17743/jaes.2018.0043 


\title{
Mixed Analytical-Numerical Filter Design for Optimized Electronic Control of Line Source Arrays
}

\author{
FLORIAN STRAUBE, ${ }^{1}$ AES Associate Member, FRANK SCHULTZ, ${ }^{1}$ AES Member, \\ (florian.straube@tu-berlin.de) (frank.schultz@tu-berlin.de) \\ MICHAEL MAKARSKI, ${ }^{2}$ AES Member, AND STEFAN WEINZIERL ${ }^{1}$ \\ (michael.makarski@ifaa-akustik.de) \\ (stefan.weinzierl@tu-berlin.de) \\ ${ }^{1}$ Audio Communication Group, TU Berlin, DE-10587 Berlin, Germany \\ ${ }^{2}$ Institute for Acoustics and Audio Technique, DE-52146 Würselen, Germany
}

\begin{abstract}
Line source arrays (LSAs) are used for large-scale sound reinforcement aiming at the synthesis of homogeneous sound fields for the whole audio bandwidth. The deployed loudspeaker cabinets are rigged with different tilt angles and/or are electronically controlled in order to provide the intended coverage of the audience zones and to avoid radiation towards the ceiling, reflective walls or residential areas. In this contribution a mixed analytical-numerical approach, referred to as line source array venue slice drive optimization (LAVDO), is introduced for optimizing the individual loudspeakers' driving functions. This method is compared to numerical optimization schemes including least-squares and multiobjective goal attainment approaches. For two standard LSAs in straight and in curved configuration, these temporal frequency domain optimizations are performed for a typical concert venue. It is shown that LAVDO overcomes the non-smooth frequency responses resulting from numerical frequency domain approaches. LAVDO provides smooth amplitude and phase responses of the loudspeakers' driving functions that is essential for the subsequent practical finite impulse response filter design and implementation.
\end{abstract}

\section{O INTRODUCTION}

For the optimization of the curving and the electronic control of line source arrays (LSAs) for advanced largescale sound reinforcement there is no standard procedure. In practice, a pure geometric and a pure electronic wavefront shaping, as well as combinations thereof are realized [1]. For LSAs with several individually controlled, small high frequency drivers, electronic beam steering is feasible up to high audio frequencies. While the cabinets of most LSA systems are curved in addition to the electronic beam steering, the cabinets of others are rigged in a straight line.

Gain and delay are the two parameters-sometimes called excitation coefficients, feeding coefficients or driving functions - that have to be ascertained for electronic optimization of the LSA radiation. They are typically computed separately for each frequency. These two parameters can be mathematically considered as the amplitude and phase of a complex frequency-dependent driving function.

The established Wavefront Sculpture Technology [2] consists of five criteria on how to create a homogeneous wavefront based on geometric shaping of uniformly driven LSAs [3]. It does not include criteria for finding optimized driving functions for the individual loudspeakers. As a result, appropriate coupling and equalization filters are still adjusted manually [4].

Least-squares optimization algorithms with Tikhonov regularization are frequently used in sound field synthesis applications for determining the loudspeakers' driving functions. They include methods such as the loudspeaker weight energy method, which minimizes the spatial average error between the desired and the synthesized sound field imposing an energy constraint on the loudspeaker weights [5].

In the field of multi-zone sound field synthesis, the minimization of the spatial average error often comes with a loudspeaker weight energy-like constraint and is linked to the minimization of the sound pressure in the dark zone. Alternatively, the error minimization is linked to the acoustic contrast control, i.e., maximizing the ratio of the average sound pressure in the bright and dark zones [6]. The bright/dark zones correspond to the audience/nonaudience zones in large-scale sound reinforcement problems.

In the context of LSA optimization, a least-squares algorithm has been extended by spatial weighting for the control 
positions and measures to make the driving robust against small deviations of the LSA characteristics [7]. Also, genetic algorithms have been used for calculating appropriate loudspeaker driving signals [8]. Wave field synthesis as an approach for wavefront shaping within a target listening plane using a contour of loudspeakers has also been proposed as a large-scale sound reinforcement technique [9-11].

Recent, mostly proprietary, software such as Martin Audio Display [12], EAW Resolution 2 [13], d\&b ArrayCalc [14], and AFMG FIRmaker [15] offer (numerical) optimization schemes. These approaches yield considerable improvements with respect to homogeneous audience coverage and/or avoidance of high side lobe energy compared to manually adjusted setups. However, the algorithms and their parametrization are rarely publicly documented except in [16-19]. In the cited papers, a multiobjective goal attainment optimization approach [20] is used, i.e., a vector of multivariable functions incorporating the loudspeakers' driving functions as variables are optimized. The objective vector contains the optimization goals, such as the error between the desired and the generated sound field, or the ratio of the sound pressures in the audience and in the nonaudience zones, with specified weights for the different objectives.

The present article is focused on the optimization of the individual driving signals of LSAs with subsequent finite impulse response (FIR) filter design. We therefore introduce a mixed analytical-numerical optimization scheme, referred to as line source array venue slice drive optimization (LAVDO). We compare this method to the least-squares approach with Tikhonov regularization applied in [5] and a multiobjective goal attainment approach applied in $[18,19]$. LAVDO is meant to overcome the non-smooth frequency responses resulting from numerical frequency domain approaches. Its features, i.e., combining far-field considerations and the multiobjective goal attainment approach for curve fitting optimization across broad frequency ranges, as well as the subsequent practical FIR filter design, are examined.

For optimizing the sound fields at the selected control positions in the venue slice, cf., Fig. 2, goals such as flat magnitude responses, maximizing the sound pressure levels (SPLs) at all audience positions and minimizing the SPLs at all non-audience positions, are pursued. Acoustic simulations based on the complex-directivity point source model [21, 22] including measured far-field loudspeaker directivity data provide the basis for an evaluation of the different optimization approaches.

The article is organized as follows. In Sec. 1 the chosen LSA configuration and the selected exemplary concert venue are presented first of all for convenient variable introduction. The complex-directivity point source model for sound field prediction is briefly recalled in Sec. 2. Two numerical methods and the introduced LAVDO_-for optimizing the driving functions of the individual loudspeakers of LSAs - as well as the method for designing practical FIR filters from the calculated driving functions are described in Sec. 3.

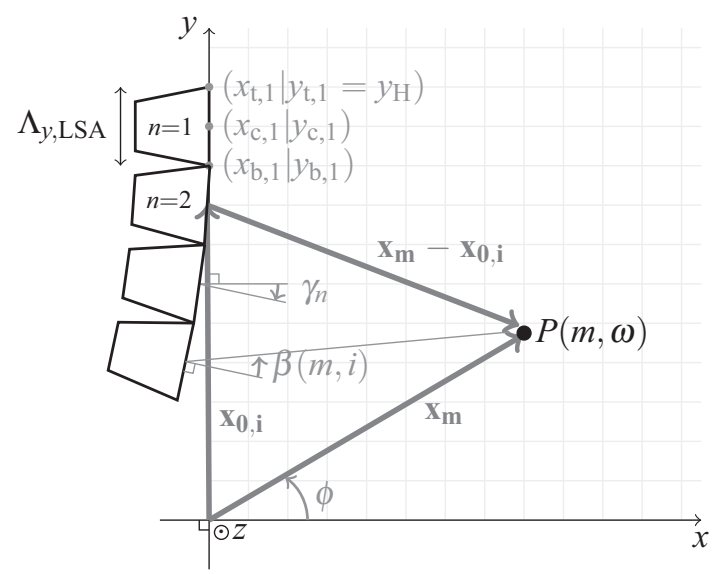

Fig. 1. Schematic sketch of the LSA setup under discussion. A total of $N=4$ LSA cabinets with the individual height $\Lambda_{y, \mathrm{LSA}}=$ $0.346 \mathrm{~m}$ is used.

In Sec. 4, the three aforementioned optimization schemes are applied for intended wavefronts based on the control positions in the venue slice. For one optimization goal, the calculated driving functions are presented. The optimization results are discussed and compared in Sec. 5, leading to the conclusion in Sec. 6.

\section{SIMULATION SETUP}

An LSA setup in straight and in curved configuration is examined for a typical concert venue with audience and non-audience sections given within the $x y$-plane. The sources are characterized by geometric attributes such as the number of cabinets, cabinet dimensions, and tilt angles, as well as electric and acoustic attributes such as the loudspeaker directivity, sensitivity, and the crossover functions. The venues are specified by the source and the receiver positions that are classified as either audience or non-audience positions.

\subsection{Line Source Array Setup}

The LSA setup and the geometry under discussion are schematically depicted in Fig. 1 for calculating the sound pressure $P(m, \omega)$ at the angular frequency $\omega$ and the $m$-th receiver position characterized by the vector $\mathbf{x}_{\mathbf{m}}$. A total of $N=4$ LSA cabinets with $n=1,2,3,4$ is deployed in order to allow for a clear comparison of the optimization methods. The front grille's height $\Lambda_{y, \mathrm{LSA}}$ of a single LSA cabinet is set to $0.346 \mathrm{~m}$ resulting in an overall LSA length of $1.384 \mathrm{~m}$. Fixing the mounting height of the LSA, the top position of the first LSA cabinet is $y_{\mathrm{H}}=2 \Lambda_{y, \text { LSA }}$, i.e., the LSA is approximately centered around $y=0$. The individual tilt angles are $\gamma_{n}$, and $\mathbf{x}_{\mathbf{0}, \mathbf{i}}$ denotes the front grille center position vector of the $i$-th LSA driver. For the straight LSA $\gamma_{n}=0$ holds for all cabinets. For the curved array, the optimized tilt angles $\left(\gamma_{1}, \gamma_{2}, \gamma_{3}, \gamma_{4}\right)^{\mathrm{T}} \approx\left(6.2^{\circ}, 6.6^{\circ}, 7.2^{\circ}\right.$, $\left.8.1^{\circ}\right)^{\mathrm{T}}$ were calculated by applying the polygonal audience line curving approach 2 (PALC2) [23] to the considered venue geometry in Fig. 2. The vectors $\mathbf{x}_{\mathbf{t}, \mathbf{n}}, \mathbf{x}_{\mathbf{c}, \mathbf{n}}$, and $\mathbf{x}_{\mathbf{b}, \mathbf{n}}$ 


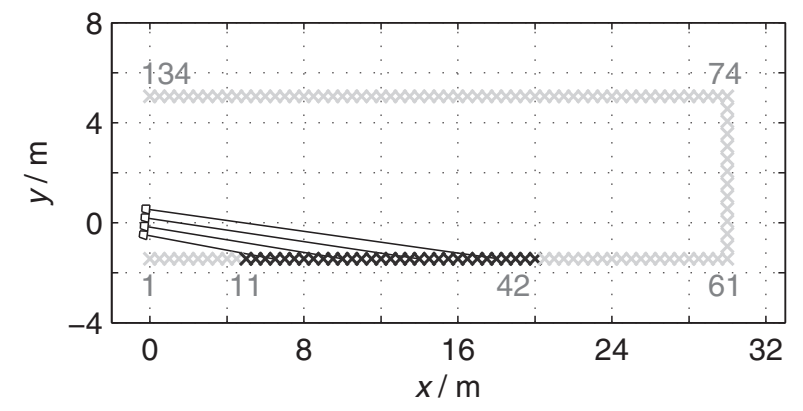

Fig. 2. Venue slice under discussion within the $x y$-plane with audience (black) as well as non-audience/avoid (gray) positions and selected index numbers (change of audience/avoid zone and/or polygonal line's section angle) from $M$ receiver positions.

define the top, center, and bottom position of the $n$-th LSA cabinet, respectively. By the radiation angle $\beta(m, i)$, the source-receiver configuration is specified for the $i$-th source and the $m$-th receiver position. $\phi$ denotes the far-field radiation angle. Further detailed information on the geometric configuration can be found in [24].

Built from three-way cabinets in this article, the chosen LSA consists of $V_{\mathrm{LF}}=1, V_{\mathrm{MF}}=2$, and $V_{\mathrm{HF}}=5$ vertically stacked, individually controlled drivers per cabinet for the low, mid, and high frequency band (LF, MF, HF). Twelveinch, 6.5- inch, and 2.64-inch drivers are used for LF, MF, and HF, respectively. Thus, the LSA consists of a total of $V \cdot N$ sources with $i=1,2, \ldots, V \cdot N$ for each frequency band. The so called Active Radiating Factor amounts to 0.61 for LF, 0.72 for MF, and 0.97 for HF following [25].

Using measured loudspeaker directivity data for the sound field predictions and optimizations, the frequency dependent sensitivities $S_{\mathrm{LF}}(i, \omega), S_{\mathrm{MF}}(i, \omega)$, and $S_{\mathrm{HF}}(i, \omega)$ are also measured in order to obtain realistic sound pressure values for vertical radiation. For the frequency band crossover, linear-phase Linkwitz-Riley filters with the transition frequencies of $f_{\mathrm{LF}, \mathrm{MF}}=400 \mathrm{~Hz}$ and $f_{\mathrm{MF}, \mathrm{HF}}=$ $1.1 \mathrm{kHz}$ are applied.

\subsection{Venue Geometry}

A standard concert venue with audience (bright zone, high SPL) and non-audience (dark zone, low SPL) sections is modeled by a venue slice representation in the $x y$-plane considering vertical LSA radiation, cf., Fig. 2. It consists of one audience line $(15 \mathrm{~m})$ and four non-audience lines and resembles an elementary sound reinforcement setting for easily interpretable optimization results.

A distance of $0.5 \mathrm{~m}$ for adjacent receiver positions was chosen, which corresponds to $M=134$ considered receiver positions $(m=1,2, \ldots, M)$. The receiver positions are composed of $M_{\mathrm{a}}$ audience positions from the set $\mathcal{M}_{\mathrm{a}}$ and $M_{\text {na }}$ non-audience positions from the set $\mathcal{M}_{\text {na }}$ with $M=M_{\mathrm{a}}$ $+M_{\mathrm{na}}$. They are characterized by the position vectors $\mathbf{x}_{\mathrm{m}}=$ $\left(x_{m}, y_{m}, 0\right)^{\mathrm{T}}$ and are numbered counterclockwise starting from the position directly beneath the LSA (index 1, cf., Fig. 2). Note that the receiver positions have to cover at least \pm 90 degree of the main LSA radiation direction referring to the center of a straight LSA in order to avoid severe side lobes resulting from numerical optimization schemes.

\section{CALCULATION MODEL}

The sound field prediction and optimization are based on the complex-directivity point source model [21, 22]. For predicting the sound pressure

$$
P(m, \omega)=\sum_{i=1}^{i=V \cdot N} G(m, i, \omega) D(i, \omega)
$$

[21, Eq. (5)], [22, Eq. (11)] is used. Eq. (1) considers the sources $i$ with a total of $N$ LSA cabinets each equipped with $V$ loudspeakers in a specified frequency band (LF, MF, HF). $P(m, \omega)$ denotes the sound pressure with the angular frequency $\omega$ at the $m$-th receiver position characterized by the vector $\mathbf{x}_{\mathbf{m}} \cdot G(m, i, \omega)$ terms the acoustic transfer function from the $i$-th source to the $m$-th receiver position

$$
G(m, i, \omega)=p_{0} 10^{\frac{S(i, \omega)}{20}} R(\beta(m, i), \omega) \frac{\mathrm{e}^{-\mathrm{j} \frac{\omega}{c}\left|\mathbf{x}_{\mathbf{m}}-\mathbf{x}_{0, \mathbf{i}}\right|}}{\left|\mathbf{x}_{\mathbf{m}}-\mathbf{x}_{\mathbf{0}, \mathbf{i}}\right|} .
$$

It is composed of (i) a frequency-band specific far-field radiation pattern $R(\beta(m, i), \omega)$ for the radiation angle $\beta(m$, i) with the angular frequency $\omega$, (ii) the ideal point source wave propagation $\frac{\mathrm{e}^{-\mathrm{j}} \frac{\mathrm{\omega} \mid}{c} \mathbf{x}_{\mathrm{m}}-\mathbf{x}_{0, \mathrm{i}} \mid}{\left|\mathbf{x}_{\mathrm{m}}-\mathbf{x}_{0, \mathrm{i}}\right|}$ with the speed of sound $c$ and $\mathrm{j}^{2}=-1$, (iii) the reference sound pressure $p_{0}=2 \cdot 10^{-5} \mathrm{~Pa}$ in air, and (iv) the loudspeaker sensitivity $S(i, \omega)$ specifying the SPL in $1 \mathrm{~m}$ distance for $1 \mathrm{~W}$ electrical input power.

Utilizing Eq. (1) for the sound field prediction, the calculated sound fields result from the superposition of the impact of the sources $i$. The impact of each source is characterized by the source-receiver propagation characteristicsdescribed by the acoustic transfer function $G(m, i, \omega)$-and by its signal characteristics, i.e., the signal input as well as the electronic filters affecting the input of each sourcedescribed by the driving function $D(i, \omega)$.

$$
D(i, \omega)=D_{\text {in }}(i, \omega) D_{\text {opt }}(i, \omega) D_{\text {xo }}(\omega)
$$

of the $i$-th source at the angular frequency $\omega$ consists of the signal input $D_{\text {in }}(i, \omega)$, the complex optimization filter $D_{\text {opt }}(i, \omega)$ - which is to be found and implemented as an FIR filter - and the complex frequency band crossover/high/low pass filters $D_{\text {xo }}(\omega)$. Gain and delay are mathematically considered as the amplitude and phase of these complex functions.

Eq. (1) reads $\mathbf{p}(\omega)=\mathbf{G}(\omega) \mathbf{d}(\omega)$ in matrix notation, accounting for all receiver positions $M$ at a single angular frequency $\omega$ with (i) $\mathbf{p}(\omega)$ denoting the $(M \times 1)$ vector of sound pressures for all considered receiver positions $\mathbf{x}_{\mathbf{m}}$, (ii) $\mathbf{G}(\omega)$ denoting the $(M \times V \cdot N)$ acoustic transfer function matrix from all drivers $i$ to all receiver positions $m$, and (iii) $\mathbf{d}(\omega)$ denoting the $(V \cdot N \times 1)$ vector of the complex driving functions for all drivers $i$ per angular frequency $\omega$. Then, for a desired sound field $\mathbf{p}_{\text {des }}(\omega)$ at the considered evaluation positions $\mathbf{x}_{\mathbf{m}}$

$$
\mathbf{p}_{\mathrm{des}}(\omega)=\mathbf{G}(\omega) \mathbf{d}(\omega)
$$


has to be solved for the driving functions $\mathbf{d}(\omega)$. Since typically $M>V \cdot N$ in the discussed application, i.e., the number of evaluation positions is larger than the number of individual sources, an ill-posed inverse problem must be analyzed $[26,27]$. For the numerical optimization schemes considered in this article, the optimization is performed separately for each frequency.

\section{ELECTRONIC CONTROL METHODS FOR SOUND FIELD OPTIMIZATION}

In the following section different numerical optimization schemes are discussed which were applied to sound reinforcement with LSAs. Alternatively, the mixed analyticalnumerical approach LAVDO is introduced. Their optimization results are examined in Sec. 4. These approaches operate in the frequency domain. They are only focused on the optimization of the loudspeakers' driving functions with subsequent FIR filter generation, without optimization of the LSA curving. The practical FIR filter design from the calculated driving functions is considered in this section as well.

\subsection{Least-Squares Optimization with Tikhonov Regularization}

In [5] the least-squares optimization with Tikhonov regularization of the driving functions is termed loudspeaker weight energy according to the considered constraint. In order to solve Eq. (4) with respect to the loudspeaker weights, the objective function to be minimized reads

$$
\begin{gathered}
\min _{\mathbf{d}(\omega)}\left\|\mathbf{G}(\omega) \mathbf{d}(\omega)-\mathbf{p}_{\mathrm{des}}(\omega)\right\|_{2}^{2} \\
\text { subject to: }\left\|\mathbf{d}_{\text {opt }}(\omega)\right\|_{2}^{2} \leq D_{\max }^{2}
\end{gathered}
$$

denoting the squared Euclidean norm $\|\cdot\|_{2}^{2}$ [28, Eq. (3.2.13)] and the constraint $D_{\max }^{2}$ with $D_{\max , \mathrm{dB}}=$ $10 \lg D_{\max }^{2}$ as the limit for the summed squares of the driving functions' absolute values, i.e., the total energy of the driving functions (cf., [5, Eq. (1)]). The solution is known as

$$
\mathbf{d}\left(\omega, \lambda_{\text {reg }}\right)=\left[\mathbf{G}(\omega)^{\mathrm{H}} \mathbf{G}(\omega)+\lambda_{\text {reg }} \mathbf{I}_{V \cdot N}\right]^{-1} \mathbf{G}(\omega)^{\mathrm{H}} \mathbf{p}_{\mathrm{des}}(\omega),
$$

with the regularization parameter $\lambda_{\text {reg }}$. The Hermitian is denoted by ${ }^{\mathrm{H}}$ and $\mathbf{I}_{V N}$ is a $(V \cdot N \times V \cdot N)$ identity matrix. Taking $D_{\max }^{2}$ into account, $\lambda_{\text {reg }}$ can be found by means of singular value analysis and using Newton's method, cf., [5, Sec. II. B/C].

\subsection{Multiobjective Goal Attainment Approach}

The multiobjective goal attainment optimization approach [20] is applied in [18, 19]. Its equation is (cf., [20, Eq. (1)] for a generalization)

$$
\begin{array}{cc}
\min _{\zeta, \mathbf{d}(\omega)} \zeta \\
\text { such that: } & \mathbf{F}[\mathbf{d}(\omega)]-\mathbf{w} \zeta \leq \mathbf{F}^{*}[\mathbf{d}(\omega)] \\
\text { subject to: } & \left|D_{\mathrm{opt}}(i, \omega)\right| \leq D_{\mathrm{opt}, \max }(\omega) \quad \forall i \\
& \left|D_{\mathrm{opt}}(i, \omega)\right| \geq D_{\mathrm{opt}, \min }(\omega) \quad \forall i
\end{array}
$$

with two exemplary constraints for the maximum $D_{\text {opt,max }}(\omega)$ and the minimum value $D_{\text {opt, } \min }(\omega)$ of the amplitudes $\left|D_{\text {opt }}(i, \omega)\right|$ of the individual driving functions and with the vector of objective functions

$$
\mathbf{F}[\mathbf{d}(\omega)]=\left(\begin{array}{c}
F_{1}[\mathbf{d}(\omega)] \\
F_{2}[\mathbf{d}(\omega)]
\end{array}\right)=\left(\begin{array}{c}
E(\omega) \\
L_{p, \mathrm{a}, \mathrm{na}}(\omega)
\end{array}\right)
$$

that shall incorporate two goals in this case. The vector of objective functions comprises the frequency dependent absolute amplitude error [24, Eq. (16)]

$$
E(\omega)=\left\|\underset{m \in \mathcal{M}_{\mathrm{a}}}{\mathbf{p}(\omega)}-\underset{m \in \mathcal{M}_{\mathrm{a}}}{\mathbf{p}_{\mathrm{des}}}(\omega)\right\|_{2}^{2}
$$

between the desired and the generated sound field in the audience zone as well as the frequency dependent ratio of the mean squared sound pressures of the audience and the non-audience zone, given as level

$$
L_{p, \text { a,na }}(\omega)=10 \lg \left(\frac{\frac{1}{M_{\mathrm{a}}}\left\|\underset{m \in \mathcal{M}_{\mathrm{a}}}{\mathbf{p}(\omega)}\right\|_{2}^{2}}{\frac{1}{M_{\mathrm{na}}}\left\|\underset{m \in \mathcal{M}_{\mathrm{na}}}{\mathbf{p}(\omega)}\right\|_{2}^{2}}\right),
$$

cf., [24, Eq. (18)]. The latter measure corresponds to the so called acoustic contrast [6, Eq. (16)] established in multizone sound field synthesis.

In general, the optimization in Eq. (7) is aimed at minimizing the parameter $\zeta$, i.e., the difference of the objective functions $\mathbf{F}[\mathbf{d}(\omega)]$ and their design goals $\mathbf{F}^{*}[\mathbf{d}(\omega)]$. Optimizing for one objective typically results in impairing other objectives when using multiobjective goal attainment optimization approaches. Including the weighting vector $\mathbf{w}$ in the optimization allows for balancing the different objectives. In combination with the minimization parameter, the product $\mathbf{w} \zeta$ entails that the design goals do not necessarily have to be rigidly met.

\subsection{Mixed Analytical-Numerical Approach/LAVDO}

Referred to as line source array venue slice drive optimization, abbreviated LAVDO, due to the application of controlling positions from the venue slice, a mixed analytical-numerical approach is introduced here. It is based on the idea of finding meaningful initial solutions analytically and refining them numerically (here using the multiobjective goal attainment optimization approach) in a subsequent step.

Different optimization goals can be included, such as maximizing the ratio of the obtained sound pressures in the audience and in the non-audience zones, flat frequency responses at all audience positions, as well as maximizing the SPLs at all audience positions. In contrast to the aforementioned "complex" optimizations, the amplitudes and the phases are calculated separately using LAVDO in order to clearly distinguish between beam width and beam steering effects. Therefore, the analytically determined initial solutions are found by means of far-field considerations for straight as well as curved LSA configurations. 


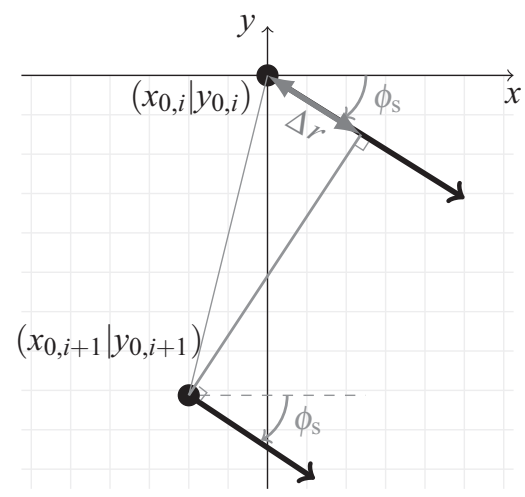

Fig. 3. Sketch of the far-field beam steering for a curved array used for finding an initial optimization solution for the LAVDO approach. It is visualized for two sources and for a beam steered downwards by $\phi_{\mathrm{s}}$.

The motivation for LAVDO originates from intending smooth amplitude and smooth phase frequency responses. This smoothness is not ensured using numerical-only approaches with driving functions optimized separately for different frequencies. Smooth frequency responses are necessary for the practical FIR filter design in order to obtain moderate filter lengths and in order to deal with uncertainties of the measurement data and of the geometric source-receiver configuration. Due to the initial solutions of LAVDO, physically unfeasible solutions that may be found by numerical-only optimization schemes do not have to be eliminated by successive post-processing. The approach results in a restricted solution space and exhibits low computational load.

For the initial solutions, the (group) delays of the individual loudspeakers are sought iteratively for every considered frequency at first, i.e., with respect to $\mathrm{e}^{\mathrm{j}[\omega t+\varphi(i, \omega)]}$ in the time domain. Hence, each angle $\varphi(i, \omega)$ of the complex driving function of the $i$-th loudspeaker is calculated depending on the steering angle $\phi_{\mathrm{s}}$, so that $D_{\text {opt }}(i, \omega)=\mathrm{e}^{\mathrm{j} \varphi(i, \omega)}$.

For a curved LSA configuration, cf., Fig. 3,

$$
\begin{aligned}
\frac{\varphi(i, \omega)}{\omega}= & \frac{\Delta r}{c} \\
= & \sin \left(\phi_{\mathrm{s}}-\frac{\pi}{2}+\arctan \left[\frac{y_{0, i+1}-y_{0, i}}{x_{0, i+1}-x_{0, i}}\right]\right) \\
& \times \frac{\sqrt{\left(x_{0, i+1}-x_{0, i}\right)^{2}+\left(y_{0, i+1}-y_{0, i}\right)^{2}}}{c} \\
= & \frac{\sin \phi_{\mathrm{s}}\left|y_{0, i+1}-y_{0, i}\right|-\cos \phi_{\mathrm{s}}\left|x_{0, i+1}-x_{0, i}\right|}{c}
\end{aligned}
$$

holds for downward beam steering and $\varphi(i+1, \omega)=0$, i.e., only considering the delay difference between two sources by Eq. (11), cf., [29, Sec. 7.8] for a straight array.

According to the different optimization goals,

(i)

$$
\max _{\phi_{\mathrm{s}}} L_{p, \mathrm{a}, \mathrm{na}}\left(\omega, \phi_{\mathrm{s}}\right)
$$

is set for maximizing the ratio of the mean squared sound pressures in the audience and in the non-audience zones, see Eq. (10) for the definition of $L_{p, \text { a,na }}(\omega)$. If flat frequency responses are desired at all audience positions,

$$
\max _{\phi_{\mathrm{s}}}\left\|\underset{m \in \mathcal{M}_{\mathrm{a}}}{\|}\right\| \|_{\infty}
$$

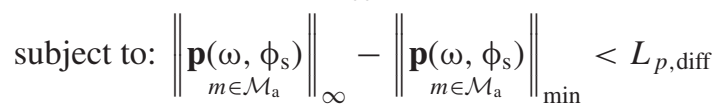

is computed denoting the maximum norm $\|\cdot\|_{\infty}[28, \mathrm{Eq}$. (3.2.13)] and defining a minimum norm

$$
\left\|\underset{m \in \mathcal{M}_{\mathrm{a}}}{\mathbf{p}\left(\omega, \phi_{\mathrm{s}}\right)}\right\|_{\min }=\min _{m \in \mathcal{M}_{\mathrm{a}}}\left|P_{m}\left(\omega, \phi_{\mathrm{s}}\right)\right| .
$$

Eq. (13) incorporates the constraint that the difference of the maximum SPL and the minimum SPL does not exceed

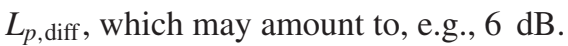

Subsequently, if smooth SPL responses are desired at all audience positions, a global magnitude $D_{\mathrm{g}}(\omega)$ is determined for each frequency. This global magnitude is equal for all sources per frequency band and it does not exceed $0 \mathrm{~dB}$. It is computed by adapting $D_{\mathrm{g}}(\omega)$ iteratively regarding the respective calculated maximum SPLs at all audience positions, so that $D_{\text {opt }}(i, \omega)=D_{\mathrm{g}}(\omega) \mathrm{e}^{\mathrm{j} \varphi(i, \omega)}$. Hence, to achieve a smooth amplitude response, the maximum approved SPL can either be chosen based on a reference frequency, e.g., $f=1 \mathrm{kHz}$, or on a fixed reference SPL, e.g., $L_{p}=90 \mathrm{~dB}_{\mathrm{SPL}}$.

These initial solutions for the individual driving functions are then smoothed with respect to frequency. In a next step, the amplitudes and unwrapped phases are fitted separately to predetermined curves, such as polynomials

$$
\left|D_{\text {opt }}(i, \omega)\right|=\sum_{k=0}^{k=K} \nu_{\mathrm{mag}, k, i}\left(\frac{\omega}{f_{\mathrm{s}}}\right)^{k}
$$

of the $K$-th degree for the magnitudes with the $k$-th curve parameter $\nu_{\text {mag, } k, i}$ for the $i$-th source and the sampling frequency $f_{\mathrm{s}}$. For the phase, the $K$-th order Fourier series

$$
\begin{aligned}
\arg \left\{D_{\mathrm{opt}}(i, \omega)\right\} & =\sum_{k=0}^{k=K} \nu_{\cos , k, i} \cos \left(k \nu_{\text {fund }, i} \frac{\omega}{f_{\mathrm{s}}}\right) \\
& +\sum_{k=0}^{k=K} \nu_{\sin , k, i} \sin \left(k \nu_{\text {fund }, i} \frac{\omega}{f_{\mathrm{s}}}\right),
\end{aligned}
$$

with the curve parameters $\nu_{\cos , k, i}, \nu_{\sin , k, i}$, and $\nu_{\text {fund, } i}$ is used for the considered angular frequency $\omega$ and the $i$-th LSA source. This smoothing limits the solution space regarding different frequencies.

Compared to fitting the FIR filter coefficients directly, a further subsequent processing step, i.e., the filter design, is necessary but the magnitudes and the phases can still be dealt with independently which is one key factor for magnitude and phase-controlled optimization filters. This approach ensures that the effects of magnitude and phase optimization can be physically understood and allows for excluding unfeasible solutions. It also allows to control the dynamic range of the magnitudes and the group delay of the filters separately. The conversion from a logarithmicallyspaced to the required linearly-spaced frequency vector is not processed at this stage so that the number of considered 
frequencies is kept low with a frequency distribution that is rather perceptually motivated, but it has to be adjusted in a later stage.

Applying the multiobjective goal attainment optimization approach, the final solution is computed, again separately for the driving functions' amplitudes and phases. In contrast to the method in Sec. 3.2, the optimizations are not performed by determining the amplitudes and/or phases for each driver at a discrete frequency. Instead, the curve parameters $\nu$ of the fitting functions are sought for all evaluated frequencies ensuring smooth amplitude and phase responses. The smoothness is maintained and while the difference between the goals and the obtained sound fields and driving functions may be improved for several frequencies, it may be impaired for some other frequencies. Forming the $(V \cdot N \times \Psi)$ matrix $\mathbf{D}\left(\nu_{i}\right)$ with the number $\Psi$ of evaluated frequencies that contains the driving functions $\mathbf{d}(\omega)$ for all frequencies, Eq. (7) is for $\nu_{i} \in\left\{\nu_{\operatorname{mag}, k, i}, \nu_{\cos , k, i}\right.$, $\left.\nu_{\text {sin, } k, i}, \nu_{\text {fund, } i}\right\}$ rewritten as

$$
\begin{array}{cc} 
& \min _{\zeta, v_{i} \zeta} \zeta \mathbf{F}^{*}\left[\mathbf{D}\left(v_{i}\right)\right] \\
\text { such that: } & \mathbf{F}\left[\mathbf{D}\left(\nu_{i}\right)\right]-\mathbf{w} \zeta \leq D_{\text {opt,max }}(\omega) \quad \forall \omega \\
\text { subject to: } & \left|D_{\mathrm{opt}}(i, \omega)\right| \leq D_{\mathrm{opt} \text { min }}(\omega) \quad \forall \omega .
\end{array}
$$

The vector of objective functions $\mathbf{F}\left[\mathbf{D}\left(v_{i}\right)\right]$ contains the goals according to Eqs. (12) and (13), respectively. Note that the optimization constraints can be freely chosen in this approach.

\subsection{Driving Filter FIR Design}

Following the optimization of the individual driving functions, the calculated amplitude and phase frequency responses are implemented by means of FIR filters for practical application to loudspeakers. The filters are designed with the frequency sampling method [30]. Hence, a linearly-spaced frequency vector is required for the inverse discrete Fourier transform. Since it is reasonable to use a logarithmically-spaced frequency vector for the optimizations with respect to computational efficiency and human perception, this vector has to be converted prior to the filter design.

For all optimizations in this article, a logarithmicallyspaced frequency, $\frac{1}{12}$ octave resolution vector with $f_{\text {opt, } \min }=100 \mathrm{~Hz}$ and $f_{\text {opt, } \max }=20 \mathrm{kHz}$ is used. Utilizing measured loudspeaker data for the sound field prediction, the frequency vector is slightly modified by picking those frequencies from the linearly-spaced measurement frequency vector that are nearest to the ones of the logarithmically-spaced optimization vector. This "quasi" logarithmically-spaced vector proved to be more practical with respect to the results and the computational effort than interpolating the linearly-spaced measurement frequency vector in order to obtain a purely logarithmically-spaced one in advance.

\section{OPTIMIZATION RESULTS}

In this section the three sound field optimization approaches from Sec. 3 are compared based on simulations

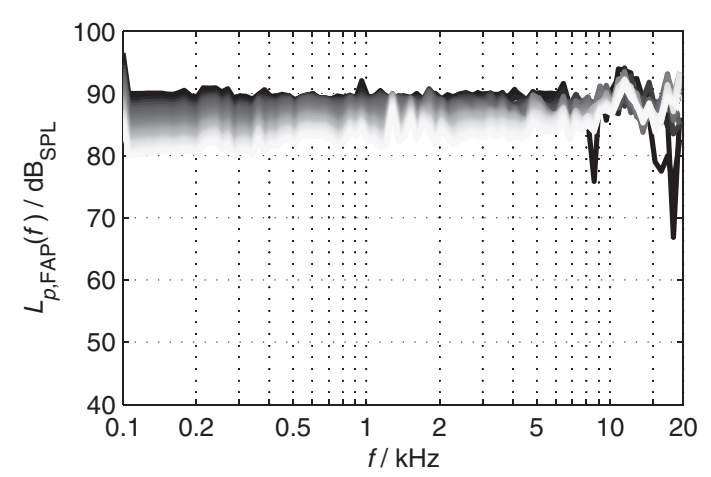

(a) SPL frequency responses at all audience positions

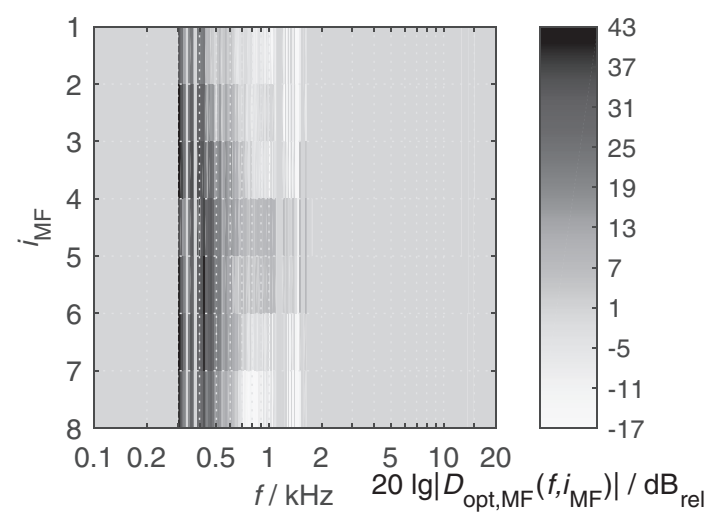

(b) driving function index plot

Fig. 4. Exemplary least-squares optimization with Tikhonov regularization for the straight LSA from Sec. 4.1. SPL frequency responses at all audience positions (a) and driving function index plot (b) for all mid frequency way sources are shown. In the SPL frequency responses at all audience positions, the curves become brighter from the first to the last audience position.

considering the audience and the non-audience positions in the vertical radiation plane, cf., Fig. 2. The desired sound field depends on the required characteristics in the audience zone, such as flat magnitude responses, a fixed SPL loss per distance doubling or maximum SPL at all positions, as well as in the non-audience zone, such as minimum SPL directed towards ceilings, reflective walls, residential areas or the stage. The performance evaluation, i.e., the visualizations and the technical quality measures, is intentionally not consistently given for all approaches in this section. They are rather individually selected in order to reveal various deficiencies leading to the developed feature set of the LAVDO.

\subsection{Least-Squares Optimization with Tikhonov Regularization}

For the least-squares optimization with Tikhonov regularization from Sec. 3.1, the sound field $P_{\mathrm{des}}(m, \omega)$ with $3 \mathrm{~dB}$ level loss per distance doubling is desired. A target SPL of $90 \mathrm{~dB}_{\mathrm{SPL}}$ at the first receiver position within the audience zone $(m=11)$ is requested for the venue in Fig. 2.

In Fig. 4 optimization results are shown for a straight LSA configuration and the parameters $D_{\max } \mathrm{dB}=30 \mathrm{~dB}$ as 
the limit for the summed squares of the driving functions' absolute values and $\lambda_{\text {reg, } 0}=1$ as initial regularization parameter. $D_{\max , \mathrm{dB}}=30 \mathrm{~dB}$ means that the limit for the summed squares of the driving functions' absolute values amounts to 1000 , i.e., possible solutions for the driving functions' magnitudes considering three sources could be for example (i) $\sqrt{(1000,0,0)}$ or (ii) $\sqrt{(1000 / 2,1000 / 2,0)}$ or (iii) $\sqrt{(1000 / 3,1000 / 3,1000 / 3)}$ which corresponds approximately to (i) $(30,-\infty,-\infty) \mathrm{dB}$ and (ii) $(27,27,-\infty)$ $\mathrm{dB}$, and (iii) $(25,25,25) \mathrm{dB}$, respectively. These examples reveal that the upper limit of the single driving functions' magnitudes cannot be directly specified by $D_{\max } \mathrm{dB}$ and that the lower limit is not defined at all. The visualizations comprise the SPL frequency responses at all audience positions and the driving function index plot representing the magnitudes over frequency that have to be applied to the individual sources $i$ in order to obtain the optimized sound field [24].

In Fig. 4 it can be observed that the desired wavefront can be well synthesized up to ca. $10 \mathrm{kHz}$ neglecting the level response at the first audience position when using the leastsquares algorithm. The driving function index plot reveals that the individual sources, depicted for the mid frequency way in this case, are rather unevenly controlled with respect to the frequency and to the sources, i.e., the frequency responses are not smooth. As the driving functions are calculated separately for each frequency, a variation greater than the chosen $30 \mathrm{~dB}$ is possible between different frequencies. Note that the group delays of the driving functions are not visualized as they also suffer from inadequate frequency smoothness analogue to the magnitudes, cf., the results in [24].

\subsection{Multiobjective Goal Attainment Approach}

The multiobjective goal attainment optimization approach from Sec. 3.2 is applied using

$$
\begin{aligned}
\mathbf{F}[\mathbf{d}(\omega)]=\left(\begin{array}{c}
E(\omega) \\
L_{p, \mathrm{a}, \mathrm{na}}(\omega)
\end{array}\right) & =\left(\begin{array}{c}
10^{-6} \mathrm{~Pa}^{2} \\
15 \mathrm{~dB}_{\text {rel }}
\end{array}\right) \\
D_{\text {opt,min,dB }}(\omega) & =-12 \mathrm{~dB}_{\text {rel }} \\
D_{\text {opt,max }, \mathrm{dB}}(\omega) & =0 \mathrm{~dB}_{\text {rel }} \\
\mathbf{w} & =(1,-1 / 0.1)^{\mathrm{T}}
\end{aligned}
$$

for a straight LSA configuration. According to Eq. (18), minimum and maximum values are assigned as constraints for the magnitudes of the driving functions, a level difference of $15 \mathrm{~dB}$ between the sound pressures in the audience and in the non-audience zone is demanded per frequency, and the minimization of the absolute error between the desired and the synthesized sound field is given a higher weight than the maximization of the acoustic contrast. As in Sec. 4.1 the desired sound field is set to exhibit $3 \mathrm{~dB}$ SPL loss per distance doubling. In Fig. 5 the SPL frequency responses at all audience positions, the driving function index plot, and the acoustic contrast $L_{p, \text { a,na }}(f)$ are shown for this exemplary optimization.

In the SPL frequency responses at all audience positions, it can be seen that the desired sound field is well synthesized up to ca. $1 \mathrm{kHz}$. This frequency approximately

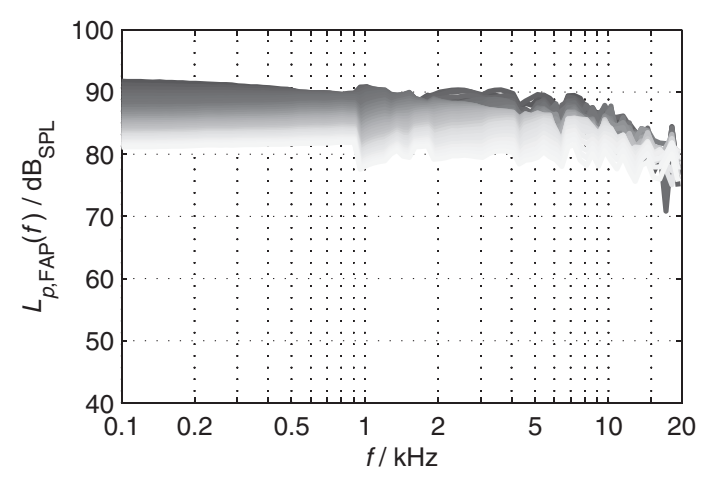

(a) SPL frequency responses at all audience positions

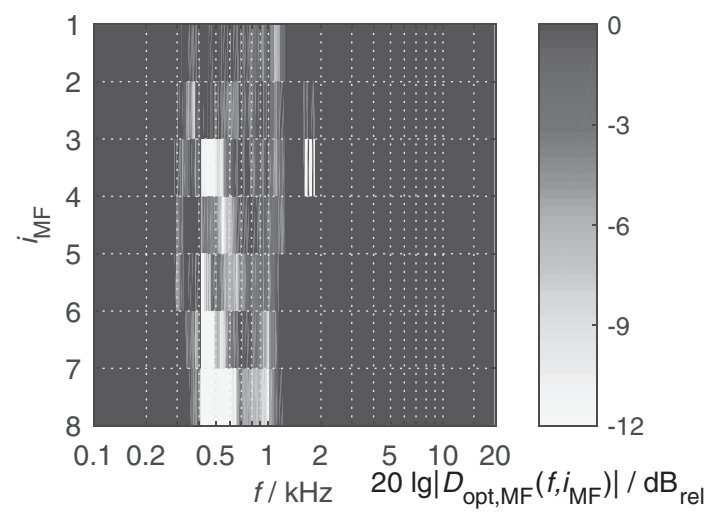

(b) driving function index plot

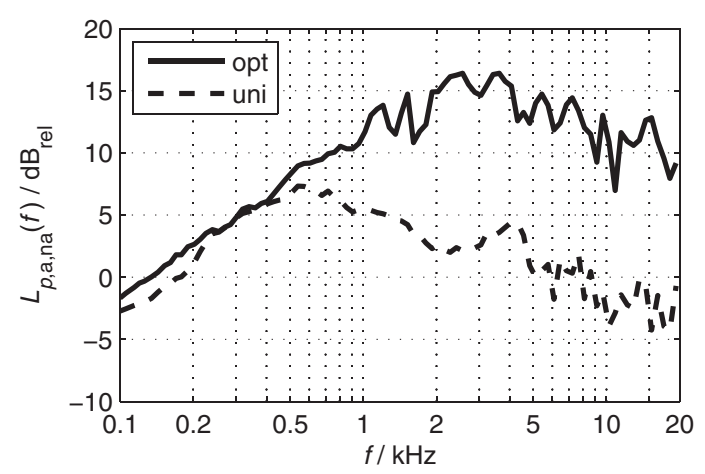

(c) acoustic contrast $L_{p, \text { a,na }}(f)$

Fig. 5. Exemplary multiobjective goal attainment optimization for the straight LSA from Sec. 4.2. SPL frequency responses at all audience positions (a), driving function index plot (b) for all MF sources, and the acoustic contrast $L_{p, \text { a,na }}(f)$ (c) are shown. In the SPL frequency responses at all audience positions, the curves become brighter from the first to the last audience position.

coincides with the MF-HF-crossover frequency. Above that frequency, the SPL loss per distance doubling exceeds $3 \mathrm{~dB}$ but the frequency responses are quite flat with a decrease of the maximum SPL above ca. $8 \mathrm{kHz}$. The behavior in the MF-HF-crossover range is caused by the transition and combination from/of the MF driving function to/and the $\mathrm{HF}$ driving function optimization with a different amount of involved loudspeakers. These effects could be decreased by additional post-processing that was not considered for this plot in order to reveal this weakness. 
In the main working range of the mid frequency loudspeakers from ca. $300 \mathrm{~Hz}$ up to ca. $1.5 \mathrm{kHz}$, the driving function index plot reveals insufficient level smoothness but the range of the values is restricted to the constrained maximum and minimum value. Compared to the driving functions resulting from the least-squares optimization in Sec. 4.1, the deviation of the magnitudes between the different sources is considerably decreased, yielding a more feasible and economical driving load. Plotting $L_{p, \text { ana }}(f)$, the desired increase of the acoustic contrast is clearly noticeable for frequencies for which the LSA is capable of directional coverage.

\subsection{Mixed Analytical-Numerical Approach/LAVDO}

Applying the mixed analytical-numerical approach from Sec. 3.3, referred to as LAVDO, for optimizing the individual loudspeakers' driving functions, there is no need for defining the exact desired sound field analytically. The desired sound field is initially given by the uniformly driven LSA radiation. Each magnitude of the individual sources' driving functions is constrained to $D_{\text {opt, max, } \mathrm{dB}}(\omega)=0 \mathrm{~dB}_{\text {rel }}$ for the initial solutions and the numerical refinements.

For each parameter set, a straight and a curved LSA configuration are examined. Polynomials of the first degree and Fourier series terms up to the 7 th order serve as fitting functions for the numerical refinements of the driving functions' amplitudes and unwrapped phases, respectively. For frequencies $f>200 \mathrm{~Hz}$, the optimizations are performed.

Two goals are pursued: (i) achieving a maximum ratio of the average sound pressures in the audience and in the nonaudience zones, cf., Eq. (12), and (ii) achieving a constant SPL with a tolerance of $\pm 3 \mathrm{~dB}$ at all audience positions, cf., Eq. (13). For all goals, the weighting factors for the numerical refinements are chosen to 1 for minimizations and -1 for maximizations. The objective function vector for goal (i) is written as

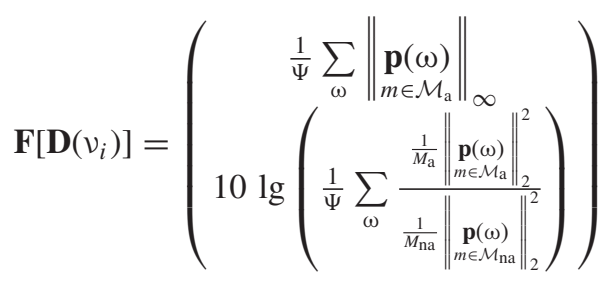

with the maximum norm $\|\cdot\|_{\infty}$. The first objective is the average of the maximum sound pressures at all audience positions over all frequencies and the second objective function is the average of the ratios of the sound pressures in the audience and non-audience zone over all frequencies, analogue to $L_{p \text {,a,na }}(\omega)$. They are set to $120 \mathrm{~dB}_{\mathrm{SPL}}$ and $24 \mathrm{~dB}_{\text {rel }}$, respectively. For goal (ii) the objective function vector reads

$$
\mathbf{F}\left[\mathbf{D}\left(v_{i}\right)\right]=\left(\begin{array}{c}
10 \lg \left(\frac{1}{p_{0}^{2} M_{\mathrm{a}}}\left\|\begin{array}{c}
\mathbf{p}\left(\omega=\omega_{\mathrm{ref}}\right) \\
m \in \mathcal{M}_{\mathrm{a}}
\end{array}\right\|_{2}^{2}\right. \\
10 \lg \left(\frac{1}{p_{0}^{2} M_{\mathrm{a}}}\left\|\begin{array}{c}
\mathbf{p}\left(\omega=\omega_{\mathrm{ref}}\right) \\
m \in \mathcal{M}_{\mathrm{a}}
\end{array}\right\|_{2}^{2}\right)-3 \mathrm{~dB}
\end{array}\right)
$$

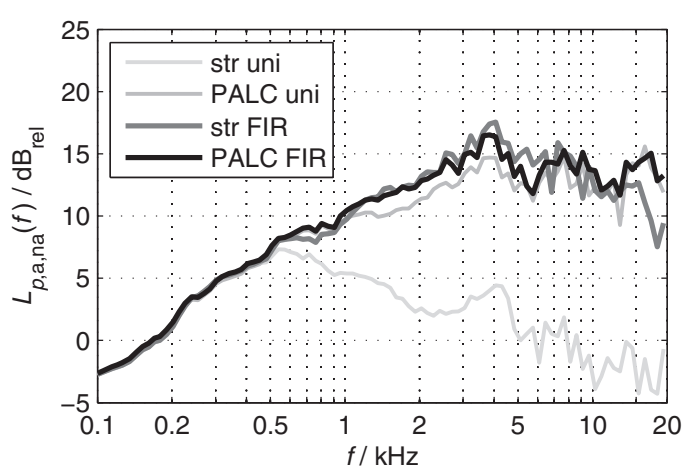

Fig. 6. $L_{p, \text { ana }}(f)$ - the level of the frequency dependent ratio of the mean squared sound pressures of the audience and the nonaudience zone - for the straight ("str") and the curved ("PALC") configuration with the tilt angles from Sec. 1.1. Both the values for the uniformly driven ("uni") as well as the optimized ("FIR") LSAs are visualized. The used goal (i) is to achieve maximum acoustic contrast, here $15 \mathrm{~dB}$.

with the reference angular frequency $\omega_{\text {ref }}=2 \pi f_{\text {ref }}$ with $f_{\text {ref }}=1 \mathrm{kHz}$. Here, objectives 1 and 2 contain the average SPLs over all audience positions at the reference frequency and the $\pm 3 \mathrm{~dB}$ tolerance.

Comparing the acoustic contrast for the case including its maximization, goal (i), cf., Fig. 6, the ratio of the mean squared sound pressures of the audience and the nonaudience zones is considerably higher for the curved and electronically controlled straight array than for the uniformly driven straight array as expected. This is valid for high frequencies for which the LSA does not radiate omnidirectionally. The PALC2 curved LSA further gains slightly increased acoustic contrast by additionally applied optimization filters. $L_{p \text {, a,na }}(f)$ does not differ substantially for the optimized straight and the optimized curved LSA configuration in this rather simple reinforcement scenario. Only small differences within a small band width are observable.

Ensuring SPL frequency responses as flat as possible, cf., SPL frequency responses at all audience positions in Fig. 7, the SPL tolerance of $\pm 3 \mathrm{~dB}$ of goal (ii) can be accomplished up to ca. $4 \mathrm{kHz}$. Above this frequency, the SPL loss from the first audience positions to the last audience positions exceeds this limit. The maximum SPLs are rather low in favor of the flat frequency responses taking the high frequency level decrease into account and not allowing gains larger than $0 \mathrm{~dB}$.

\subsection{LAVDO Driving Functions}

In Fig. 8 the transfer functions' levels and group delays are visualized for the LAVDO case from Sec. 4.3 with the goal (ii) of achieving a constant SPL with a tolerance of $\pm 3 \mathrm{~dB}$ at all audience positions. They are shown for a straight LSA configuration.

Low pass characteristics can be observed for the low as well as mid frequency way level in Fig. 8. Compensating for the decreasing SPLs with increasing frequency, high pass analogies are observable for the high frequency way levels. For the frequency transitions between the low and mid 


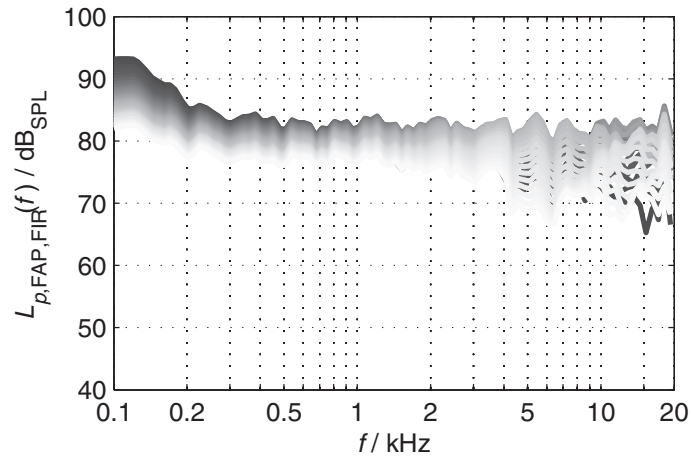

(a) straight LSA

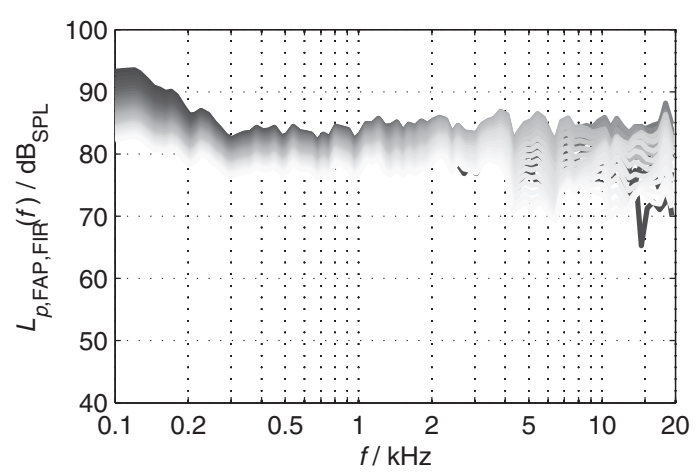

(b) curved LSA

Fig. 7. Line source array venue slice drive optimization (LAVDO) with optimization goal (ii): achieve a constant SPL with \pm 3 dB tolerance at all audience positions. SPL frequency responses at all audience positions (FAP) for the line source array driven with the calculated FIR filters for the straight (a) and curved (b) configuration with the tilt angles from Sec. 1.1 are shown. In the SPL frequency responses at all audience positions, the curves become brighter from the first to the last audience position.

frequency as well as between the mid and high frequency range, the driving functions of both frequency ways affect the SPL frequency responses at the receiver positions. The mid frequency magnitude and phase frequency response values for low frequencies-where the influence of the mid frequency cabinets are small-result from extrapolation of the mid frequency values from the frequencies where the influence of the mid frequency cabinets is significant. Except for a small low frequency range, the group delays do not exceed $6 \mathrm{~ms}$. Note that the extracted pre-delays are not shown for this optimization case.

\section{DISCUSSION}

In Table 1 the most significant characteristics of the three presented optimization methods in this article are summarized. Considering these, it shows that the least-squares algorithm with Tikhonov regularization and the multiobjective goal attainment method require extensive postprocessing and/or re-optimizations for obtaining smooth magnitude and phase frequency responses. This is due to performing the optimizations at discrete frequencies. Although it is possible to include constraints that connect nearby frequencies, the choice of a reference value is not obvious, i.e., which frequency and which amplitude and phase shall serve as the reference. Since these approaches incorporate complex optimizations, combined for the magnitudes and phases, the magnitude and phase effects cannot be clearly distinguished after the optimizations.

Comparing the mid frequency driving functions of the numerical optimization schemes in Figs. $4 \mathrm{~b}$ and $5 \mathrm{~b}$ with the driving functions of the presented mixed analyticalnumerical approach LAVDO in Fig. 8c, the deviations between the driving function magnitudes for different loudspeakers and for different frequencies can be clearly seen for the numerical methods. For LAVDO, the increased smoothness of the magnitude frequency responses in Fig. 8c is obvious considering different loudspeakers and different frequencies.
Applying the least-squares algorithm with Tikhonov regularization from Sec. 3.1 it is not possible to set constraints on all individual sources but only on several ones together. Therefore, the resulting power loads of the individual drivers must be carefully monitored. This is one considerable drawback of the deployed loudspeaker weight energy algorithm.

With the multiobjective goal attainment approach, separate optimization constraints can be selected for the individual sources and several goals can be pursued weighted according to the intended priority. This includes the practically relevant control of the driver efficiency and the driver power balance by including corresponding constraints. Utilizing the advantages of the multiobjective goal attainment method and reducing the computational costs, the presented mixed analytical-numerical approach LAVDO restricts the solution space in advance, avoiding extensive post-processing and avoiding non-smooth frequency responses.

For all driving function optimization schemes, the choice of the desired sound field or rather the intended wavefront shape is crucial. While analytically exact sound fields may be advantageous for modeled loudspeaker data, the desired sound fields should, in practice, rather be based on a feasible solution, such as, the sound fields generated by a uniformly driven LSA used here or manually pre-adjusted setups. Using measured loudspeaker data, this approach incorporates the specific radiation characteristics of the considered LSA and it does not force the magnitudes and phases to differ significantly from the measured values, i.e., the influence of the FIR filters is kept low. With a low influence of the FIR filters, the impact of the uncertainties of the measurement data and of the geometric source-receiver configuration may also be limited. This approach restricts the freedom of the optimization algorithms resulting in fewer potential solutions and also reduces the amount of physically unrealizable solutions.

For the design of practical FIR filters the chosen frequency resolution is important. A linearly-spaced frequency vector is necessary for performing the inverse discrete 


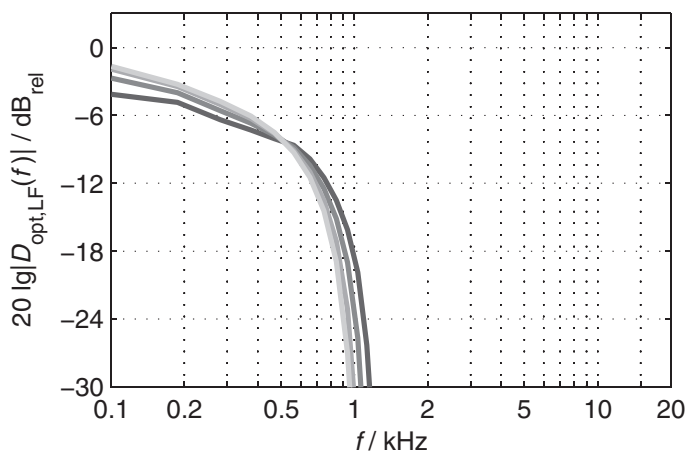

(a) LF level

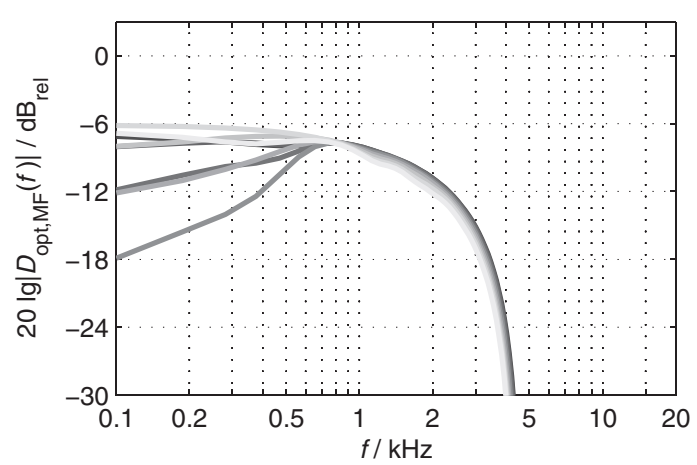

(c) MF level

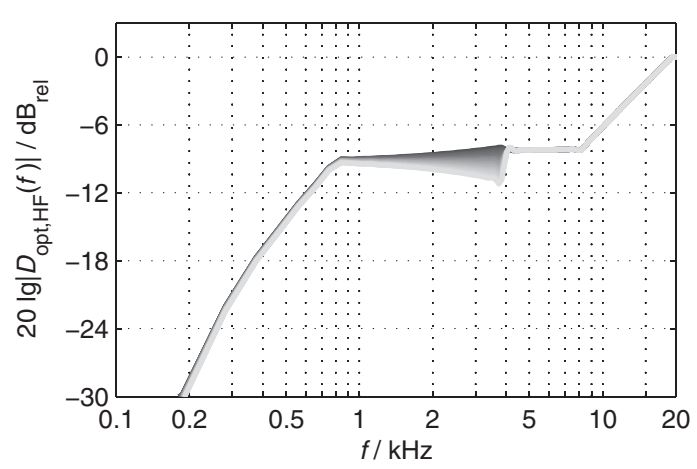

(e) HF level

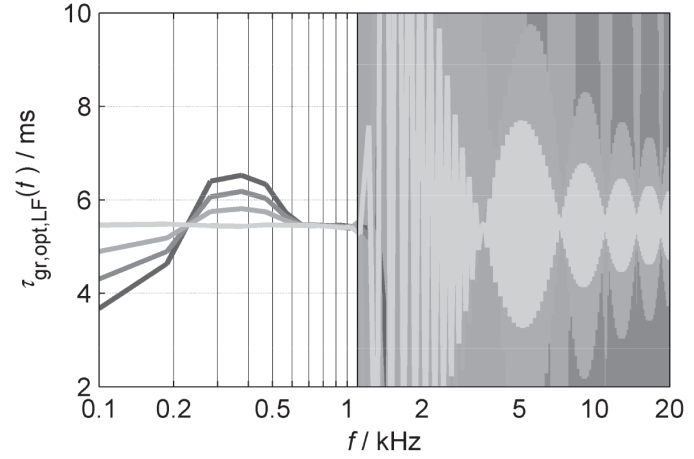

(b) LF group delay

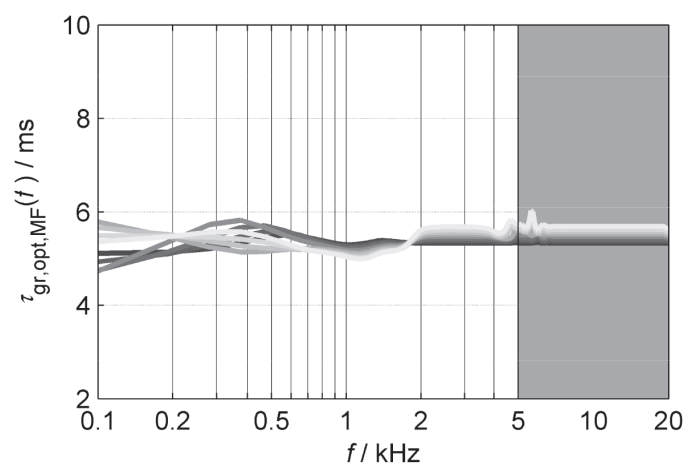

(d) MF group delay

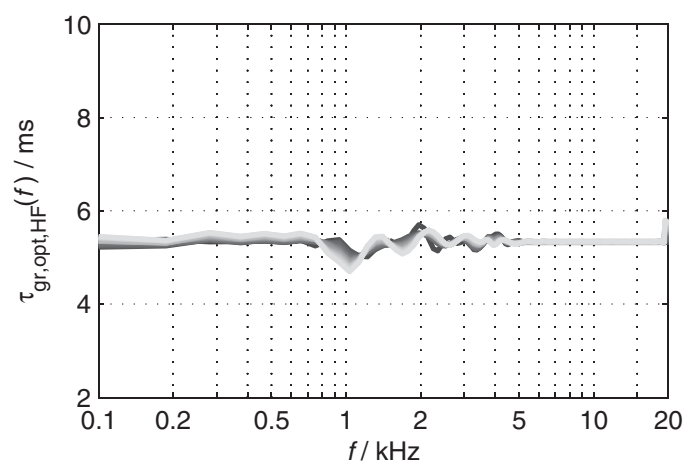

(f) HF group delay

Fig. 8. Driving functions for the line source array venue slice drive optimization (LAVDO) with straight LSA from Fig. 7, left. Level responses (a), (c), (e) and group delays (b), (d), (f) of all loudspeakers of the different frequency bands ((a), (b): low frequency sources, (c), (d): mid frequency sources, (e), (f): high frequency sources) are shown. The optimization goal (ii) is to achieve a constant SPL with $\pm 3 \mathrm{~dB}$ tolerance at all audience positions from Sec. 4.3. In the plots, the curves become brighter from the topmost to the bottommost LSA enclosure. Parts of the group delays that are irrelevant due to the working range of the different frequency bands are charted gray.

Fourier transform. Due to performance reasons with respect to the optimization schemes and due to perceptual reasons, it is convenient to cover the audio bandwidth with a logarithmically-spaced frequency vector. The logarithmically-spaced vector thus has to be converted to a linear scale before generating the FIR filter coefficients.

\section{CONCLUSION}

In this article different optimization approaches for the electronic drive of line source arrays (LSAs) are applied for exemplary sound reinforcement setups. These methods include the least-squares optimization with Tikhonov regularization, the multiobjective goal attainment approach, and the line source array venue slice drive optimization (LAVDO) that is introduced as a mixed analyticalnumerical approach in this contribution. Both straight and curved LSA configurations are examined based on measured loudspeaker directivity and sensitivity data. The optimizations are performed for audience and non-audience positions along the vertical venue slice. As a last step, the conversion from the calculated individual loudspeakers' driving functions to practical finite impulse response filters is considered. 
Table 1. Comparison of the discussed optimization methods for sound reinforcement with LSAs.

\begin{tabular}{|c|c|c|c|}
\hline optimization method & $\begin{array}{l}\text { least-squares optimization with } \\
\text { Tikhonov regularization }\end{array}$ & $\begin{array}{l}\text { multiobjective goal attainment } \\
\text { approach }\end{array}$ & $\begin{array}{l}\text { mixed analytical-numerical } \\
\text { approach (LAVDO) }\end{array}$ \\
\hline optimization goals & only one optimization goal & $\begin{array}{l}\text { several optimization goals are } \\
\text { possible }\end{array}$ & $\begin{array}{l}\text { several optimization goals } \\
\text { are possible }\end{array}$ \\
\hline optimization constraints & $\begin{array}{l}\text { separate constraints on individual } \\
\text { sources are not possible }\end{array}$ & $\begin{array}{l}\text { separate constraints on } \\
\text { individual sources are possible }\end{array}$ & $\begin{array}{l}\text { separate constraints on } \\
\text { individual sources are } \\
\text { possible }\end{array}$ \\
\hline power limitation & $\begin{array}{l}\text { no limitation of the maximum } \\
\text { tolerated electric power of the } \\
\text { individual sources }\end{array}$ & $\begin{array}{l}\text { limitation of the maximum } \\
\text { tolerated electric power of the } \\
\text { individual sources is possible }\end{array}$ & $\begin{array}{l}\text { limitation of the maximum } \\
\text { tolerated electric power } \\
\text { of the individual sources } \\
\text { is possible }\end{array}$ \\
\hline load balance & $\begin{array}{l}\text { resulting loads must be carefully } \\
\text { monitored for practically } \\
\text { feasible drive }\end{array}$ & $\begin{array}{l}\text { range of the resulting loads can } \\
\text { be easily controlled }\end{array}$ & $\begin{array}{l}\text { range of the resulting loads } \\
\text { can be easily controlled }\end{array}$ \\
\hline $\begin{array}{l}\text { frequency smoothness } \\
\text { complexity }\end{array}$ & $\begin{array}{l}\text { cannot be directly controlled } \\
\text { simple optimization scheme }\end{array}$ & $\begin{array}{l}\text { cannot be directly controlled } \\
\text { elaborate optimization scheme }\end{array}$ & $\begin{array}{l}\text { can be easily controlled } \\
\text { elaborate optimization } \\
\text { scheme }\end{array}$ \\
\hline computational efficiency & mid load & high load & low load \\
\hline
\end{tabular}

Smooth amplitude and phase frequency responses are necessary when dealing with uncertainties of the measurement data and of the geometric source-receiver configuration as well as for the FIR filter design in order to obtain moderate filter lengths. The introduced LAVDO approach ensures smooth responses as the magnitudes and phases are fitted separately to predetermined curves. Since the numerical refinement does not operate frequency-wise and since the calculation of the initial solutions is based on analytical considerations excluding physically unfeasible solutions in advance and furthermore allowing broad parameter control, extensive post-processing and/or re-optimizations can be avoided. Therefore LAVDO turns out to be more aimoriented, more robust and lower-computational than the considered least-squares optimization and the pure multiobjective goal attainment approach.

The optimization results show that the desired sound fields can be realized with straight as well as curved LSA setups. If the array is not geometrically adapted to the audience zone, small source distances are, however, necessary for grating lobe free beam steering up to the highest audio frequencies. Therefore it seems to be reasonable to find a meaningful interaction of geometric and electronic wavefront shaping using LSAs for practical realizations with respect to technical and economical considerations. The electronic influence and hence the applied optimization algorithms can be kept to a manageable level if the LSA geometry is appropriately adapted to the sound reinforcement area.

For the future, it is planned to consider additional LAVDO optimization goals and to combine these within the numerical computation in order that an absolute weighting between the different goals is possible. It is also planned to examine whether the curve fitting within the LAVDO approach should be directly based on the filter coefficients and not on the associated driving functions, i.e., combining the calculation of the driving functions and the subsequent FIR filter design to be one single stage.
Wave field synthesis based initial solutions shall be incorporated rather than initial solutions found by far-field considerations.

\section{REFERENCES}

[1] D. Scheirman, "Large-Scale Loudspeaker Arrays: Past, Present, and Future (Part Two-Electroacoustic Considerations)," presented at the AES 59th International Conference: Sound Reinforcement Engineering and Technology (2015 Jul.), conference paper 1-3.

[2] M. Urban, C. Heil, and P. Bauman, "Wavefront Sculpture Technology," J. Audio Eng. Soc., vol. 51, pp. 912-932 (2003 Oct.).

[3] M. S. Ureda, "Analysis of Loudspeaker Line Arrays," J. Audio Eng. Soc., vol. 52, pp. 467-495 (2004 May).

[4] L'Acoustics, "Training Module Variable Curvature Line Source," L'Acoustics (2016).

[5] T. Betlehem and C. Withers, "Sound Field Reproduction with Energy Constraint on Loudspeaker Weights," IEEE Trans. on Audio, Speech, and Language Processing, vol. 20, no. 8, pp. 2388-2392 (2012 Oct.).

[6] J.-W. Choi and Y.-H. Kim, "Generation of an Acoustically Bright Zone with an Illuminated Region Using Multiple Sources," J. Acoust. Soc. Am., vol. 111, no. 4, pp. 1695-1700 (2002 Apr.), doi:https://doi.org/10.1121/ 1.1456926.

[7] G. W. van Beuningen and E. W. Start, "Optimizing Directivity Properties of DSP Controlled Loudspeaker Arrays," Proc. of the Inst. of Acoustics: Reproduced Sound, vol. 22, no. 6, pp. 17-37 (2000).

[8] M. Terrell and M. Sandler, "Optimizing the Controls of a Homogenous Loudspeaker Array," presented at the 129th Convention of the Audio Engineering Society (2010 Nov.), convention paper 8159.

[9] D. de Vries, E. W. Start, and V. G. Valstar, "The Wave Field Synthesis Concept Applied to Sound Reinforcement: Restrictions and Solutions," presented at the 96th 
Convention of the Audio Engineering Society (1994 Feb.), convention paper 3812.

[10] E. W. Start, "Application of Curved Arrays in Wave Field Synthesis," presented at the 100th Convention of the Audio Engineering Society (1996 May), convention paper 4143.

[11] F. Schultz, G. Firtha, P. Fiala, and S. Spors, "Wave Field Synthesis Driving Functions for Large-Scale Sound Reinforcement Using Line Source Arrays," presented at the 142nd Convention of the Audio Engineering Society (2017 May), convention paper 9722.

[12] Martin Audio Ltd., "User Guide. Display 2.2, version 3.2," Tech. rep., https://martin-audio.com/downloads/ software/Display-2.2-User-Guide-v3.2.pdf, last seen on 2017-08-21 (2017).

[13] Eastern Acoustic Works Inc., "EAW Resolution 2. Help File," Tech. rep., http://eaw.com/docs/3_ Manuals/EAW\%20Resolution\%202\%20User\%20Guide. pdf, last seen on 2017-08-21 (2017).

[14] d\&b Audiotechnik GmbH, "ArrayCalc Simulation Software V8. ArrayProcessing Feature, Technical White Paper," Tech. rep., http://www.dbaudio.com/en/support/ downloads/category/download/4195.html, last seen on 2017-08-21 (2017).

[15] AFMG Technologies GmbH, “AFMG FIRmaker. AFMG White Paper," Tech. rep., http://www.afmgsupport.eu/SoftwareDownloadBase/FIRmaker/FIRmaker_ White_Paper.pdf, last seen on 2017-08-21 (2017).

[16] A. Thompson, "Real World Line Array Optimisation," Proc. of the Inst. of Acoustics, vol. 30, no. 6 (2008).

[17] A. Thompson, "Improved Methods for Controlling Touring Loudspeaker Arrays," presented at the 127th Convention of the Audio Engineering Society (2009 Oct.), convention paper7828.

[18] A. Thompson, J. Baird, and B. Webb, "Numerically Optimized Touring Loudspeaker Arrays-Practical Applications," presented at the 131st Convention of the Audio Engineering Society (2011 Oct.), convention paper 8511.

[19] S. Feistel, M. Sempf, K. Köhler, and H. Schmalle, "Adapting Loudspeaker Array Radiation to the Venue Using Numerical Optimization of FIR Filters," presented at the 135th Convention of the Audio Engineering Society (2013 Oct.), convention paper 8937.
[20] F. W. Gembicki and Y. Y. Haimes, “Approach to Performance and Sensitivity Multiobjective Optimization: The Goal Attainment Method," IEEE Trans. on Automatic Control, vol. 20, no. 6, pp. 769-771 (1975 Dec.).

[21] D. G. Meyer, "Computer Simulation of Loudspeaker Directivity," J. Audio Eng. Soc., vol. 32, pp. 294315 (1984 May).

[22] S. Feistel, A. Thompson, and W. Ahnert, "Methods and Limitations of Line Source Simulation," J. Audio Eng. Soc., vol. 57, pp. 379-402 (2009 Jun.).

[23] F. Straube, F. Schultz, D. A. Bonillo, and S. Weinzierl, "An Analytical Approach for Optimizing the Curving of Line Source Arrays," J. Audio Eng. Soc., vol. 66, pp. 4-20 (2018 Jan./Feb.), doi:https://doi.org/10.17743/jaes.2017.0043.

[24] F. Straube, F. Schultz, M. Makarski, S. Spors, and S. Weinzierl, "Evaluation Strategies for the Optimization of Line Source Arrays," presented at the AES 59th International Conference: Sound Reinforcement Engineering and Technology, (2015 Jul.), conference paper 2-1.

[25] F. Schultz, F. Straube, and S. Spors, "Discussion of the Wavefront Sculpture Technology Criteria for Straight Line Arrays," presented at the 138th Convention of the Audio Engineering Society (2015 May), convention paper 9323.

[26] G. A. Deschamps and H. S. Cabayan, "Antenna Synthesis and Solution of Inverse Problems by Regularization Methods," IEEE Trans. on Antennas and Propagation, vol. 20, no. 3, pp. 268-274 (1972 May).

[27] Y. Kim and P. Nelson, "Optimal Regularization for Acoustic Source Reconstruction by Inverse Methods," J. Snd. Vibr., vol. 275, no. 3, pp. 463-487 (2004 Aug.).

[28] F. W. J. Olver, D. W. Lozier, R. F. Boisvert, and C. W. Clark, NIST Handbook of Mathematical Functions, 1st ed. (Cambridge University Press, Cambridge, 2010).

[29] L. E. Kinsler, A. R. Frey, A. B. Coppens, and J. V. Sanders, Fundamentals of Acoustics, 4th ed. (Wiley, New York, 2000).

[30] A. V. Oppenheim, R. W. Schafer, and J. R. Buck, Discrete-Time Signal Processing, 2nd ed. (Prentice-Hall, New Jersey, 1999). 
THE AUTHORS

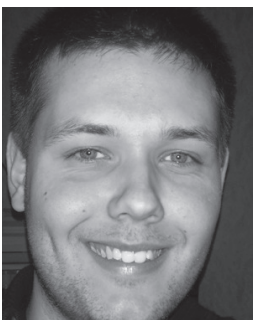

Florian Straube

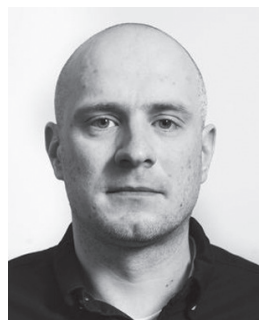

Frank Schultz

Florian Straube received the Dipl.-Ing. degree in electrical engineering/communications and information technology from TU Dresden in cooperation with Klippel $\mathrm{GmbH}$ in 2013. Since 2014 he has been working as a research associate at Audio Communication Group at TU Berlin focusing on sound field synthesis and line source array applications for sound reinforcement.

Frank Schultz received the M.Sc. degree from TU Berlin and the Dr.-Ing. degree with distinction from the University of Rostock in 2011 and 2016, respectively. Within postdoctoral research he works with the Audio Communication Group at TU Berlin and the Institute of Electronic Music and Acoustics in Graz. From 2003-2007 he worked in R\&D at Bosch Communications Systems and from 2016-2018 at sonible GmbH. Recent research interests are acoustic signal processing for and perceptual evaluation of large-scale sound reinforcement.

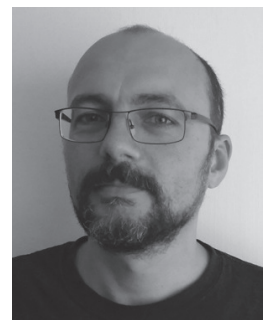

Michael Makarski

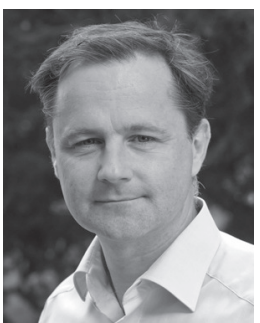

Stefan Weinzierl
Michael Makarski received the Dipl.-Ing. degree in electrical engineering in 2001 and the Dr.-Ing. degree from the RWTH Aachen University in 2006. Since 2006, he works as a freelancer with focus on the development of professional loudspeakers, acoustic measurement technique, and acoustic consulting. He is one of the founders of Four Audio GmbH \& Co. KG. Since 2007, Four Audio develops and produces DSP solutions, software, and measurement technique for professional audio applications.

Stefan Weinzierl is head of the Audio Communication Group at TU Berlin. His activities in research are focused on audio technology, virtual acoustics, room acoustics, and musical acoustics. He is coordinating a master program in audio communication and technology at TU Berlin and teaching Tonmeister students at the University of the Arts (UdK). With a diploma in physics and sound engineering and a two-year study in musicology at UC Berkeley, he received his Ph.D. from TU Berlin. He is coordinating research consortia in the field of virtual acoustics (SEACEN) and music information retrieval (ABC_DJ). 